CLINICAL STUDY

\title{
Circulating progranulin levels in women with gestational diabetes mellitus and healthy controls during and after pregnancy
}

\author{
Jelena Todoric ${ }^{1}$, Ammon Handisurya $^{2}$, Thomas Perkmann ${ }^{1}$, Bernhard Knapp ${ }^{3}$, Oswald Wagner ${ }^{1}$, Andrea Tura ${ }^{4}$, \\ Giovanni Pacini ${ }^{4}$, Harald Esterbauer ${ }^{1}$ and Alexandra Kautzky-Willer ${ }^{5}$ \\ ${ }^{1}$ Department of Laboratory Medicine, ${ }^{2}$ Division of Nephrology and Dialysis, Department of Internal Medicine III and ${ }^{3}$ Department for Biosimulation and \\ Bioinformatics, Center for Medical Statistics, Informatics and Intelligent Systems, Medical University of Vienna, Waehringer Guertel 18-20, \\ A-1090 Vienna, Austria, ${ }^{4}$ Metabolic Unit, ISIB-CNR, Padova, Italy and ${ }^{5}$ Division of Endocrinology and Metabolism, Department of Internal Medicine III, \\ Medical University of Vienna, Waehringer Guertel 18-20, A-1090 Vienna, Austria
}

(Correspondence should be addressed to J Todoric; Email: jelena.todoric@meduniwien.ac.at)

\begin{abstract}
Objective: Progranulin (PGRN) was recently introduced as a novel marker of chronic inflammatory response in obesity and type 2 diabetes capable of directly affecting the insulin signaling pathway. This study aimed to investigate the role of PGRN in gestational diabetes mellitus (GDM), which is regarded as a model for early type 2 diabetes.

Methods: PGRN serum levels were measured in 90 pregnant women (45 GDM and 45 normal glucose tolerance (NGT)). In addition, PGRN was measured during a 2-h, $75 \mathrm{~g}$ oral glucose tolerance test in 20 pregnant women (ten GDM and ten NGT) and in 16 of them post partum (ten GDM and six NGT). Results: PGRN concentrations were significantly higher in pregnant women compared with post partum levels $(536.79 \pm 31.81$ vs $241.53 \pm 8.86, P<0.001)$. Multivariate regression analyses showed a strong positive correlation of PGRN with estrogen and progesterone. The insulinogenic index, a marker of early insulin secretion, displayed a positive correlation with PGRN, both during and after pregnancy $(R=0.47, P=0.034 ; R=0.63, P=0.012)$. HbA1c and the oral glucose insulin sensitivity index showed significant post partum associations with PGRN $(R=0.43, P=0.049 ; R=-0.65$, $P=0.009$ ).

Conclusions: PGRN concentrations are markedly lower after pregnancy regardless of the gestational glucose tolerance state. PGRN levels per se do not discriminate between mild GDM and NGT in pregnant women. Therefore, the development of GDM appears to be due to impaired $\beta$-cell function that is not related to PGRN effect.
\end{abstract}

European Journal of Endocrinology 167 561-567

\section{Introduction}

Progranulin (PGRN), otherwise known as acrogranin, proepithelin, granulin-epithelin precursor, and PCcell-derived growth factor, is a widely expressed, multifunctional, high-molecular weight-secreted growth factor (1). PGRN plays a role in a variety of physiological and pathological processes including embryogenesis, inflammation, and tumor development (1). One of the best-described roles for PGRN regarding human pathologies is its capability to cause frontotemporal dementia (2). Secreted PGRN is proteolytically cleaved into subunits called granulins (GRNs) with different effects on inflammation than the precursor protein (3). While GRNs have been reported to amplify acute inflammation, PGRN was suggested as an anti-inflammatory mediator. Binding to TNF $\alpha$ receptors is one of the mechanisms by which PGRN exerts its anti-inflammatory properties, which is recently demonstrated in a model of inflammatory arthritis (4). In contrast, PGRN has been identified as a novel marker of chronic inflammation in obesity and type 2 diabetes where circulating PGRN levels were significantly elevated (5). Furthermore, PGRN correlated with BMI, total cholesterol, C-reactive protein (CRP), HbAlc, monocyte chemoattractant protein-1, and macrophage infiltration in omental adipose tissue. Visceral PGRN mRNA expression correlated positively with glucose infusion rates during the steady state of a euglycemic-hyperinsulinemic clamp (5). Recently, PGRN has been recognized as a key adipokine mediating highfat diet-induced insulin resistance. Moreover, it has been shown that PGRN directly affects insulin signaling and suppresses insulin-stimulated glucose uptake in 3T3-L1 adipocytes (6).

Gestational diabetes mellitus (GDM), defined as any degree of glucose intolerance with onset or first 
recognition during pregnancy, occurs in $\sim 7 \%$ of all pregnancies $(7,8)$. Progressive insulin resistance typically characterizes human pregnancy that begins near mid-pregnancy and reaches its peak in the third trimester as a result of increased adiposity and the insulin-desensitizing effects of placental hormones (9). Although it is known that pancreatic $\beta$-cell dysfunction is causally involved in the majority of women with GDM, its exact pathophysiology is still unclear. They are also at higher risk of developing type 2 diabetes after delivery, while their offspring are prone to childhood obesity, glucose intolerance, and type 2 diabetes later in life $(7,9)$. Thus, GDM provides an excellent opportunity to study the early pathogenesis of diabetes and can be regarded as a model for early type 2 diabetes (9).

Taking the high expression of PGRN by the human placental tissue into account, as well as its inhibitory effect on insulin signaling, it can be hypothesized that PGRN could play a role in the development of insulin resistance during pregnancy $(10,11)$. No study so far has addressed the role of circulating PGRN in pregnancy or GDM. Thus, no data are available on its potential association with other well-established GDM-related parameters. Therefore, we aimed to compare women with either normal glucose tolerance (NGT) or GDM during pregnancy and post partum looking at: i) fasting serum levels of PGRN; ii) impact of an oral glucose tolerance test (OGTT) on PGRN serum concentration; and iii) the association of serum PGRN with metabolic and hormonal parameters.

\section{Materials and methods}

\section{Subjects}

Participants for this study were recruited from the outpatient clinic of the Division of Endocrinology and Metabolism at the Medical University of Vienna via universal screening for GDM. The universal screening for GDM was performed with a 2-h, $75 \mathrm{~g}$ OGTT between the 24th and 28th weeks of gestation. For this study, healthy pregnant women were recruited before their OGTT. PGRN concentrations were measured at 0,30 , 60 , and 120 min during the OGTT in ten women with GDM and ten women with NGT matched for age and BMI in a 1:1 ratio (the OGTT group). Sixteen of these women (ten GDM and six NGT) underwent reexamination at 6-12 weeks after delivery according to the ADA guidelines (7). Additionally, in 70 women ( 35 GDM and 35 NGT), only fasting PGRN concentrations were measured. None of the women had a history of GDM or impaired glucose tolerance (IGT), and therefore, none of them had been treated for GDM at study entry. The ethics committee of the Medical University of Vienna approved this study.

\section{Biochemical analysis}

Sera were obtained by centrifugation and immediately frozen at $-80{ }^{\circ} \mathrm{C}$ until further analysis. We measured human PGRN using an ELISA for quantitative determination of PGRN in human serum, plasma, or cell culture supernatants (AdipoGen; AG-45A-0018EKKI01). Glucose, insulin, triglycerides, total cholesterol, LDL-C, HDL-C, C-peptide, CRP, HbA1c, serum creatinine, estradiol, and progesterone were quantified using tests certified for in vitro diagnostics at the Department of Laboratory Medicine of the Medical University of Vienna.

\section{Data analysis}

Insulin and C-peptide secretion during the OGTT were evaluated with the area under the insulin or C-peptide concentration curve (AUCi and AUCcp respectively). The area under the glucose concentration curve (AUCg) was calculated to reflect the total change in glucose during OGTT. AUCs were calculated using the trapezoidal rule. Fasting insulin sensitivity was assessed with the quantitative insulin sensitivity check index (QUICKI) (12), whereas dynamic insulin sensitivity was determined with the oral glucose insulin sensitivity index (OGIS) (13). QUICKI is assumed to mainly represent hepatic insulin sensitivity, while OGIS describes glucose clearance per unit change of insulin concentration, primarily representing insulin action in the peripheral tissues (muscle and fat). Total insulin secretion (TIS) from C-peptide was obtained as described previously (14). As a marker of early insulin response, we calculated the insulinogenic index (i.e. ratio of insulin increment relative to that of blood glucose 30 min after glucose load). The ability of $\beta$-cells to adapt insulin secretion to the prevailing insulin resistance is given by the product of OGIS and $\triangle \mathrm{AUCi}$, sometimes called disposition index (15), where $\triangle \mathrm{AUCi}$ is the suprabasal component of $\mathrm{AUCi}$, and by the 'adaptation index' calculated as the product of OGIS and $\Delta$ TIS (16), where $\Delta$ TIS is the suprabasal component of TIS. Both indexes provide a quantitative figure of the overall metabolic status by simultaneously accounting for insulin action and secretion.

\section{Statistical analysis}

Power analysis showed that the number of enrolled participants was adequate to evaluate the difference in PGRN concentration between NGT and GDM women during gestation and the change in PGRN concentrations across various glucose levels during OGTT in the pregnant and nonpregnant state, which were the main aims of our study. However, this study was not specifically designed and powered to examine the causal relationship between PGRN and the relatively large number of metabolic parameters that we measured. 
Data are presented as median and 25-75\% interquartile range. The Shapiro-Francia normality test was used to test continuous variables for their normal distribution. Not normally distributed data were log-transformed for the subsequent statistical analyses. Spearman rank correlations were computed to assess the relationship between variables. Multiple linear regression analyses were performed to normalize correlations for confounding variables. Differences between groups were tested by ANOVA. All statistical analyses were performed using Stata 11 (StataCorp., College Station, TX, USA). Levels of statistical significance were set at $P<0.05$.

\section{Results}

\section{PGRN and metabolic parameters in pregnancy}

Clinical, biochemical, and metabolic characteristics of women in the OGTT group and of the whole study population are given in Tables 1 and 2 respectively. Fasting PGRN concentrations did not differ significantly between NGT and GDM groups, both in women in the OGTT group and in the whole study population (Tables 1 and 2), but were markedly higher than those found in nonpregnant women (Fig. 1; $P<0.001$ ). Blood glucose levels were significantly higher in women with GDM compared with those with NGT at all time points during the OGTT (NGT vs GDM; $80.3 \pm 2.3$ vs $88.1 \pm 2.6 \mathrm{mg} / \mathrm{dl}, P<0.05$ at baseline,
$124.3 \pm 6.3$ vs $161.5 \pm 5.2 \mathrm{mg} / \mathrm{dl}, P<0.001$ at $30 \mathrm{~min}$, $135.6 \pm 7.3$ vs $186.1 \pm 11.5 \mathrm{mg} / \mathrm{dl}, P<0.01$ at $60 \mathrm{~min}$, $122.5 \pm 5.2$ vs $173.7 \pm 11.6 \mathrm{mg} / \mathrm{dl}, P<0.001$ at $90 \mathrm{~min}$, and $102.5 \pm 7.3$ vs $154.7 \pm 12.4 \mathrm{mg} / \mathrm{dl}$, $P<0.001$ at $120 \mathrm{~min}$ ) and showed a significant increase compared with the baseline (0 vs $30 \mathrm{~min}$; NGT, $P<0.001$; GDM, $P<0.001 ; 0$ vs $60 \mathrm{~min}$; NGT, $P<0.001$; GDM, $P<0.001 ; 0$ vs $90 \mathrm{~min}$; NGT, $P<0.001$; GDM, $P<0.001$; 0 vs $120 \mathrm{~min}$; NGT, $P<0.01$; and GDM, $P<0.001)$. Notably, in both NGT and GDM groups, serum PGRN concentration did not significantly change during the OGTT (0 vs 30 min; NGT, $558.9 \pm 51.4$ vs $548.4 \pm 57.8 \mathrm{ng} / \mathrm{ml}$, NS; GDM, $512.2 \pm 31.5$ vs $511.5 \pm 42.6 \mathrm{ng} / \mathrm{ml}$, NS; 0 vs $60 \mathrm{~min}$; NGT, $558.9 \pm 51.4$ vs $545.8 \pm 57.2 \mathrm{ng} / \mathrm{ml}$, NS; GDM, $512.2 \pm 31.5$ vs $501.7 \pm 36.6 \mathrm{ng} / \mathrm{ml}$, NS; 0 vs $120 \mathrm{~min}$; NGT, $558.9 \pm 51.4$ vs $551.8 \pm 51.3 \mathrm{ng} / \mathrm{ml}$, NS; and GDM, $512.2 \pm 31.5$ vs $502.2 \pm 34.1 \mathrm{ng} / \mathrm{ml}$, NS).

\section{Univariate correlations and multivariate regression analyses of basal and dynamic PGRN serum values during pregnancy}

Estrogen and progesterone displayed a highly significant positive correlation with PGRN serum levels in the whole study group $(R=0.43, P \leq 0.001$ and $R=0.53$, $P \leq 0.001$ respectively). We could not detect any correlation between PGRN and BMI, CRP, fasting glucose, fasting insulin, C-peptide, HOMA-IR, and

Table 1 Baseline characteristics and metabolic and hormonal parameters in women with GDM and NGT during pregnancy (the OGTT group). Data are presented as median and interquartile range. $P$ values: significance of differences between groups (ANOVA).

\begin{tabular}{|c|c|c|c|}
\hline & GDM $(n=10)$ & NGT $(n=10)$ & $\boldsymbol{P}$ \\
\hline Age (years) & $31.4(27-34)$ & $29.8(26-33)$ & NS \\
\hline $\mathrm{BMI}\left(\mathrm{kg} / \mathrm{m}^{2}\right)^{\mathrm{a}}$ & $27.7(25.9-31.0)$ & $26.0(24.7-29.1)$ & NS \\
\hline Systolic blood pressure $(\mathrm{mmHg})$ & $110(105-120)$ & $116.5(105-130)$ & NS \\
\hline Diastolic blood pressure $(\mathrm{mmHg})$ & $68(65-70)$ & $71(65-80)$ & NS \\
\hline Progranulin (ng/ml) & $512.2(447.9-567.1)$ & $558.9(429.1-721.4)$ & NS \\
\hline AUCpgrn (ng/ml $\times 120 \mathrm{~min})$ & 59905 (48 240-70 474) & 65951 (46 486-87 812) & NS \\
\hline Fasting glucose $(\mathrm{mg} / \mathrm{dl})$ & $88(80-95)$ & $80(76-85)$ & 0.0393 \\
\hline Fasting insulin (mU/l) & $16.0(11.3-18.8)$ & $12.2(8.3-13.2)$ & NS \\
\hline Fasting C-peptide $(\mu \mathrm{g} / \mathrm{l})$ & $2.4(1.7-2.6)$ & $1.6(1.3-1.7)$ & 0.0332 \\
\hline $\mathrm{A} 1 \mathrm{C}(\%)$ & $4.9(4.6-5.2)$ & $4.9(4.6-5.1)$ & NS \\
\hline Triglycerides (mg/dl) & 207 (161-235) & $197(150-250)$ & NS \\
\hline Total cholesterol (mg/dl) & $227(192-256)$ & $277(228-327)$ & 0.0232 \\
\hline HDL-C (mg/dl) & $66(61-75)$ & $75(59-84)$ & NS \\
\hline LDL-C (mg/dl) & $116(98-124)$ & $159(133-170)$ & 0.0214 \\
\hline CRP (mg/dl) & $0.54(0.28-0.76)$ & $0.51(0.44-0.63)$ & NS \\
\hline Creatinine (mg/dl) & $0.62(0.57-0.65)$ & $0.60(0.53-0.67)$ & NS \\
\hline Estradiol (pg/ml) & $10915(7465-13395)$ & $11161(7900-13055)$ & NS \\
\hline Progesterone (ng/ml) & $83.1(55.9-114.7)$ & $90.1(71.1-115.9)$ & NS \\
\hline AUCg (g/dl $\times 120 \mathrm{~min})$ & $19.2(17.4-20.3)^{\prime}$ & $14.2(13.6-15.5)$ & 0.0000 \\
\hline AUCi (U/I×120 min) & $15.7(11.8-19.4)$ & $8.9(7.6-10.5)$ & 0.0073 \\
\hline AUCcp $(\mathrm{ng} / \mathrm{ml} \times 120 \mathrm{~min})$ & $1334.2(1200-1708.5)$ & $1059.7(964.5-1114.5)$ & 0.0090 \\
\hline OGIS (ml/min per $\left.\mathrm{m}^{2}\right)$ & $341.5(292.1-385.6)$ & $434(413.1-459.1)$ & 0.0007 \\
\hline Insulinogenic index (pmol insulin/mmol glucose) & $126.4(94.2-174.8)$ & $126.9(71.5-200.3)$ & NS \\
\hline Disposition index & $5.15(4.75-6.29)$ & $3.87(2.67-4.43)$ & 0.0725 \\
\hline Adaptation index & $478.3(421.8-544.15)$ & $443.7(421.9-500.8)$ & NS \\
\hline
\end{tabular}

NS, nonsignificant.

${ }^{a} \mathrm{BMI}$, values at the study entry. 
Table 2 Baseline characteristics and metabolic and hormonal parameters in women with GDM and NGT during pregnancy (the whole study group). Data are presented as median and interquartile range. The significance of differences between groups were determined using ANOVA.

\begin{tabular}{|c|c|c|c|}
\hline & GDM $(n=45)$ & NGT $(n=45)$ & $\boldsymbol{P}$ \\
\hline Age (years) & $31.1(28-34)$ & $30.1(26-34)$ & NS \\
\hline BMI $\left(\mathrm{kg} / \mathrm{m}^{2}\right)^{\mathrm{a}}$ & $28.6(23.5-35.9)$ & $27.8(24.8-31.4)$ & NS \\
\hline Systolic blood pressure (mmHg) & $120(114-128)$ & $114(110-130)$ & NS \\
\hline Diastolic blood pressure $(\mathrm{mmHg})$ & $74(67-80)$ & $73(65-80)$ & NS \\
\hline Progranulin (ng/ml) & $638.3(480.9-887.0)$ & $721.4(496.7-1004.9)$ & NS \\
\hline Fasting glucose $(\mathrm{mg} / \mathrm{dl})$ & $85(79-95)$ & $81(78-86)$ & NS \\
\hline Fasting insulin (mU/l) & $11.5(8.1-17.1)$ & $11.6(8.3-19.5)$ & NS \\
\hline Fasting C-peptide $(\mu \mathrm{g} / \mathrm{l})$ & $2.4(1.6-2.6)$ & $2.1(1.3-2.8)$ & NS \\
\hline $\mathrm{A} 1 \mathrm{C}(\%)$ & $5.0(4.6-5.2)$ & $5.0(4.6-5.3)$ & NS \\
\hline Triglycerides (mg/dl) & $210(172-253)$ & $203(176-255)$ & NS \\
\hline Total cholesterol (mg/dl) & $256(215-284)$ & $270(233-309)$ & NS \\
\hline HDL-C (mg/dl) & $73(61-82)$ & $74(61-88)$ & NS \\
\hline LDL-C (mg/dl) & $139(104-164)$ & $154(121-189)$ & NS \\
\hline CRP (mg/dl) & $0.53(0.03-0.94)$ & $0.51(0.31-0.90)$ & NS \\
\hline Creatinine $(\mathrm{mg} / \mathrm{dl})$ & $0.61(0.58-0.68)$ & $0.64(0.57-0.65)$ & NS \\
\hline Estradiol (pg/ml) & $11075(7569-15455)$ & $12730(8700-16795)$ & NS \\
\hline Progesterone (ng/ml) & $74.1(55.9-117.8)$ & $104.9(71.1-162.6)$ & NS \\
\hline
\end{tabular}

NS, nonsignificant.

${ }^{\mathrm{a}} \mathrm{BMI}$, values at the study entry.

HbA1c. In the OGTT group, AUCpgrn showed a significant positive correlation with the insulinogenic index that describes the first phase of the insulin response following the glucose load $(R=0.47$, $P=0.034)$. Other OGTT-derived dynamic parameters like AUCg, AUCi, AUCcp, OGIS, and disposition index were not significantly correlated with basal PGRN and AUCpgrn.

Multiple regression models adjusted for age, BMI, and pregnancy week revealed that PGRN was closely related to estrogen and progesterone in the whole study group $(t=2.68, P=0.009 ; t=2.66, P=0.019)$. In the OGTT group, the association between AUCpgrn and the insulinogenic index remained significant.

\section{Post partum reexamination}

Women with prior GDM and women with prior NGT showed no difference concerning metabolic parameters except in the dynamic AUCi during the OGTT, which was marginally increased in women with prior GDM (Table 3). Insulin sensitivity in dynamic conditions measured by OGIS was not different between the groups at the post partum visit. Furthermore, there was no difference in serum PGRN levels between women with prior GDM and prior NGT at the post partum visit. Of note, we found a significant positive correlation between fasting insulin $(R=0.69, P=0.001 ; R=0.56, P=0.02)$ and C-peptide $(R=0.46, \quad P=0.048 ; \quad R=0.61$, $P=0.011$ ) with both fasting PGRN and AUCpgrn values respectively. Moreover, there was a borderline significant positive correlation between fasting PGRN and HbAlc $(R=0.43, P=0.049)$ and a negative correlation with QUICKI $(R=-0.63, P=0.006)$, indicating a negative impact of PGRN on insulin sensitivity. OGTT-derived dynamic parameters AUCi, AUCcp, as well as the disposition index and insulinogenic index were positively correlated with AUCpgrn $(R=0.60, P=0.018$, Fig. $2 A ; R=0.58, P=0.021$, Fig. 2B; $R=0.53, P=0.041 ; R=0.63, P=0.012$, Fig. 2C), whereas OGIS showed a significant negative correlation with AUCpgrn $(R=-0.65, P=0.009$, Fig. 2D). In multiple linear regression analyses adjusted for age, BMI, and pregnancy week, fasting PGRN remained positively related to fasting insulin and C-peptide levels $(t=4.60, \quad P<0.001 ; \quad t=2.12$, $P=0.049)$ and negatively correlated with QUICKI $(t=-2.67, P<0.019)$. Dynamic PGRN (AUCpgrn)

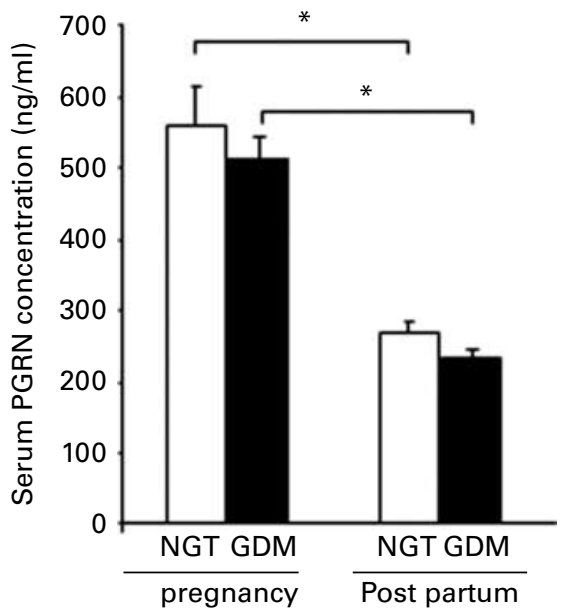

Figure 1 PGRN serum concentration measured in women with NGT and GDM during pregnancy and post partum (the OGTT group). PGRN serum concentrations were significantly higher during pregnancy compared with the levels at the post partum visit in both NGT and GDM women. ${ }^{*} P<0.001$. 
Table 3 Baseline characteristics and metabolic and hormonal parameters in women with GDM and NGT that underwent an OGTT post partum. Data are presented as median and interquartile range. The statistical significance of differences between the study groups was determined by ANOVA.

\begin{tabular}{|c|c|c|c|}
\hline & GDM $(n=10)$ & NGT $(n=6)$ & $P$ \\
\hline Age (years) & $31.9(27-35)$ & $30.2(27-33)$ & NS \\
\hline BMI $\left(\mathrm{kg} / \mathrm{m}^{2}\right)^{\mathrm{a}}$ & $27.9(26.1-31.2)$ & $26.6(25-29.6)$ & NS \\
\hline Systolic blood pressure $(\mathrm{mmHg})$ & $106(95-120)$ & $118(110-125)$ & NS \\
\hline Diastolic blood pressure (mmHg) & $65(60-70)$ & $73(65-80)$ & NS \\
\hline Progranulin (ng/ml) & $234.3(201.6-261.2)$ & $268.6(241.9-292.9)$ & NS \\
\hline AUCpgrn (ng/ml × $120 \mathrm{~min})$ & 27338 (23 819-30 143) & 29335 (24 990-33 470) & NS \\
\hline Fasting glucose (mg/dl) & $92(82-101)$ & $82(81-87)$ & NS \\
\hline Fasting insulin (mU/l) & $11.5(7.2-13.6)$ & $9.0(4.9-10.8)$ & NS \\
\hline Fasting C-peptide $(\mu \mathrm{g} / \mathrm{l})$ & $1.9(1.5-2.6)$ & $1.3(0.8-1.9)$ & NS \\
\hline $\mathrm{A} 1 \mathrm{C}(\%)$ & $5.3(5.1-5.6)$ & $5.2(5.1-5.4)$ & NS \\
\hline Triglycerides (mg/dl) & $111(56-182)$ & $77(68-91)$ & NS \\
\hline Total cholesterol (mg/dl) & $203(183-215)$ & $204(175-235)$ & NS \\
\hline HDL-C (mg/dl) & $62(46-72)$ & $65(51-71)$ & NS \\
\hline LDL-C (mg/dl) & $110(100-144)$ & $129(113.4-136)$ & NS \\
\hline $\mathrm{CRP}(\mathrm{mg} / \mathrm{dl})$ & $0.22(0.16-0.29)$ & $0.22(0.13-0.28)$ & NS \\
\hline Creatinine (mg/dl) & $0.80(0.75-0.9)$ & $0.79(0.71-0.83)$ & NS \\
\hline Estradiol (pg/ml) & $37(28-38)$ & $66(28-74)$ & NS \\
\hline Progesterone (ng/ml) & $0.8(0.6-0.9)$ & $0.4(0.3-0.5)$ & NS \\
\hline AUCg $(\mathrm{g} / \mathrm{dl} \times 120 \mathrm{~min})$ & $15.9(14.4-19.6)$ & $13.0(12.9-16.0)$ & NS \\
\hline AUCi $(U / I \times 120 \mathrm{~min})$ & $9.1(7.4-10.3)$ & $5.4(3.9-6.1)$ & 0.046 \\
\hline AUCcp $(\mathrm{ng} / \mathrm{ml} \times 120 \mathrm{~min})$ & $1131.4(858-1369.5)$ & 777.5 (610.5-919.5) & NS \\
\hline OGIS $\left(\mathrm{ml} / \mathrm{min}\right.$ per $\left.\mathrm{m}^{2}\right)$ & $408.2(370.3-436.8)$ & $476.8(441.4-526.2)$ & NS \\
\hline $\begin{array}{l}\text { Insulinogenic index } \\
\text { (pmol insulin/mmol glucose) }\end{array}$ & $132.8(88.0-150.6)$ & $109.0(60.9-139.2)$ & NS \\
\hline Disposition index & $3.57(2.92-4.06)$ & $2.50(1.98-2.33)$ & NS \\
\hline Adaptation index & $475.6(371.7-554.9)$ & 366.7 (288.7-426.4) & NS \\
\hline
\end{tabular}

NS, nonsignificant.

${ }^{a} \mathrm{BMI}$, values at the post partum visit.

remained positively correlated with AUCi, AUCcp, and the insulinogenic index $(t=2.21, P=0.049 ; t=2.21$, $P=0.049 ; t=2.78, P=0.017 ; t=2.28, P=0.041)$ and showed a negative association with OGIS $(t=-2.46$; $P=0.032)$.

\section{Discussion}

Our results showed marked elevations in fasting serum PGRN levels during gestation. PGRN serum levels were positively correlated with estrogen and progesterone and multiple regression models showed a significant influence of these two hormones on PGRN serum concentrations. This observation is in line with previous reports suggesting that PGRN is a steroid-regulated growth factor and mediator of mitogenic effects of estrogens during breast cancer development (17) as well as with a study demonstrating PGRN induction and upregulation by exogenous estrogen in the neonatal hypothalamus (18). Moreover, it has been hypothesized that PGRN may be causally involved in the estrogen-triggered mitogenic effects in adult brain areas still capable of neurogenesis (19). To our knowledge, no data are available on potential correlations of progesterone with serum PGRN levels. A single report described that progesterone-mediated changes throughout embryonic implantation in the mink uterus are closely linked to a rise in PGRN levels (20). Furthermore, this study also reported that the rise in PGRN levels corresponded to cell proliferation, remodeling, and angiogenesis during placenta establishment (20). Therefore, the higher PGRN concentrations in our pregnant women compared with their values at the post partum visit most likely reflect the higher estrogen and progesterone concentrations during pregnancy.
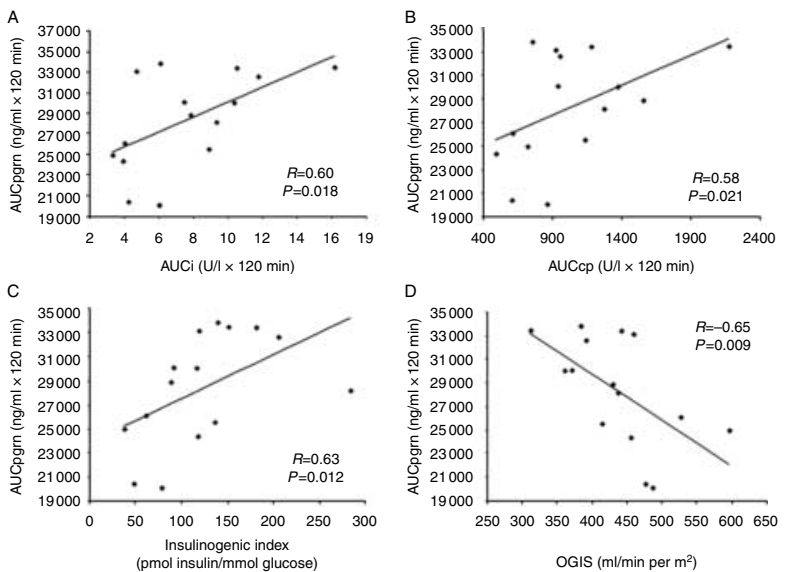

Figure 2 Correlation analysis in women that underwent OGTT post partum. (A, B, C and D) AUCpgrn was directly correlated with $A \cup C i$, AUCcp, and insulinogenic index, whereas it showed a negative relationship with OGIS. 
Hyperglycemia induced after an oral glucose load has been shown to increase circulating concentrations of several inflammatory mediators, an effect that is more pronounced in patients with impaired glucose regulation (21). In this context, our aim was to test the impact of an OGTT on PGRN serum levels in pregnant woman with GDM and NGT. We showed that acute hyperglycemia following the glucose challenge did not alter serum PGRN concentrations. Furthermore, we could not observe any differences in serum PGRN levels between women with GDM and in NGT. Interestingly, Tonjes et al. (22) have shown that PGRN levels between NGT and IGT subjects start to differ only with a certain stage of glucose intolerance. This might explain our failure to detect differences in PGRN serum levels in our groups as both GDMassociated glucose intolerance and the glucose challenge during an OGTT test are of low severity and/or duration. As demonstrated previously (5), PGRN was positively associated with chronic hyperglycemia $(\mathrm{HbAlc})$, but these associations were limited to the post partum state as we could not observe similar associations in pregnant women.

GDM women tend to have a greater insulin resistance than normal pregnant women, and the majority of them appear to have $\beta$-cell dysfunction that occurs on a background of chronic insulin resistance (12). Nonetheless, it has also been demonstrated that women with GDM, despite a reduced $\beta$-cell function during late pregnancy (23), have a greater insulin secretory response than NGT $(24,25)$. Our subjects were no exception; however, their pancreatic response was not high enough to fully compensate for the prevailing insulin resistance (demonstrated by the low insulin sensitivity). In fact, both the disposition and the adaptation indices tended to be higher in GDM without, however, reaching statistical significance, whereas the insulinogenic index showed similar values between the two groups.

Considering that PGRN showed dependent correlation with insulinogenic index, which is an indicator of early insulin secretion, we cannot rule out the possibility that PGRN may be involved in the regulation of $\beta$-cell function during pregnancy. However, our overall results suggest that PGRN plays a rather minor role in the pathophysiology of mild GDM.

AUCpgrn in women that underwent an OGTT post partum showed a significant correlation with parameters of insulin secretion: fasting insulin, fasting C-peptide, AUCi, AUCcp, disposition index, and insulinogenic index. The fasting PGRN concentration was also significantly positively correlated with fasting insulin and C-peptide levels in multiple linear regression analyses. Therefore, it may be hypothesized that insulin is an independent determinant of PGRN concentration in the nonpregnant state. The stronger association between glucose tolerance and insulin sensitivity parameters with PGRN in the nonpregnant state suggests that there are additional factors associated with pregnancy that may modify PGRN concentration. This study also found a strong negative correlation of fasting PGRN and AUCpgrn with indicators of insulin sensitivity such as QUICKI and OGIS. These results are in agreement with recently published data demonstrating that PGRN induces insulin resistance both in vivo and in vitro and directly impairs insulin signaling downstream of insulin receptors (6). Potentially, our observations suggest a predictive role of PGRN for insulin resistance at least in the nonpregnant state. The mild form of GDM in our women is one of the limitations of this study. We cannot exclude the possibility that women with a more severe form of GDM could show significantly higher PGRN levels than mild GDM patients. The limitations of this study also include the relatively small sample size of the OGTT group and the likelihood of type 1 errors due to multiple testings. Therefore, larger studies are necessary to further validate our observations.

In summary, our results showed that serum PGRN concentrations decrease in women after pregnancy, irrespective of their gestational glucose tolerance status. Although our study could not identify a causal role for PGRN in GDM occurrence and severity, the positive correlation of PGRN with HbAlc and parameters of insulin secretion as well as its negative correlation with OGIS after pregnancy suggests a possible role for PGRN in the development of post partum type 2 diabetes.

\section{Declaration of interest}

The authors declare that there is no conflict of interest that could be perceived as prejudicing the impartiality of the research reported.

\section{Funding}

This work was supported by a grant from the Jubilaeumsfond of the Austrian Nationalbank (OeNB 13244), an unrestricted grant from Takeda, and a research grant of the Vienna Science and Technology Fund (project WWTF/LSO7-058).

\section{Author contribution statement}

J Todoric carried out experiments, analyzed data, wrote, and revised the manuscript. A Handisurya carried out experiments, reviewed. and edited the manuscript. T Perkmann researched the data. B Knapp analyzed the data, reviewed, and edited the manuscript. O Wagner reviewed and edited the manuscript. A Tura and G Pacini analyzed the data, performed mathematical calculations, reviewed, and edited the manuscript. A Kautzky-Willer researched the data, reviewed, and edited the manuscript and takes responsibility for the contents of the article. $\mathrm{H}$ Esterbauer analyzed the data, reviewed, and edited the manuscript.

\section{References}

1 Liu CJ. Progranulin: a promising therapeutic target for rheumatoid arthritis. FEBS Letters 2011585 3675-3680. (doi:10.1016/ j.febslet.2011.04.065) 
2 Cruts M \& Van Broeckhoven C. Loss of progranulin function in frontotemporal lobar degeneration. Trends in Genetics $2008 \mathbf{2 4}$ 186-194. (doi:10.1016/j.tig.2008.01.004)

3 Zhu J, Nathan C, Jin W, Sim D, Ashcroft GS, Wahl SM, Lacomis L, Erdjument-Bromage H, Tempst P, Wright CD \& Ding A. Conversion of proepithelin to epithelins: roles of SLPI and elastase in host defense and wound repair. Cell 2002111 867-878. (doi:10.1016/S0092-8674(02)01141-8)

4 Tang W, Lu Y, Tian QY, Zhang Y, Guo FJ, Liu GY, Syed NM, Lai Y, Lin EA, Kong L, Su J, Yin F, Ding AH, Zanin-Zhorov A, Dustin ML, Tao J, Craft J, Yin Z, Feng JQ, Abramson SB, Yu XP \& Liu CJ. The growth factor progranulin binds to TNF receptors and is therapeutic against inflammatory arthritis in mice. Science 2011 332 478-484. (doi:10.1126/science.1199214)

5 Youn BS, Bang SI, Kloting N, Park JW, Lee N, Oh JE, Pi KB, Lee TH, Ruschke K, Fasshauer M, Stumvoll M \& Bluher M. Serum progranulin concentrations may be associated with macrophage infiltration into omental adipose tissue. Diabetes $2009 \mathbf{5 8}$ 627-636. (doi:10.2337/db08-1147)

6 Matsubara T, Mita A, Minami K, Hosooka T, Kitazawa S, Takahashi K, Tamori Y, Yokoi N, Watanabe M, Matsuo E, Nishimura O \& Seino S. PGRN is a key adipokine mediating high fat diet-induced insulin resistance and obesity through IL-6 in adipose tissue. Cell Metabolism 201215 38-50. (doi:10.1016/ j.cmet.2011.12.002)

7 American Diabetes Association. Gestational diabetes mellitus. Diabetes Care 200427 (Suppl 1) S88-S90. (doi:10.2337/diacare. 27.2007.S88)

8 American Diabetes Association. Diagnosis and classification of diabetes mellitus. Diabetes Care 201134 (Suppl 1) S62-S69. (doi:10.2337/dc11-S062)

9 Buchanan TA \& Xiang AH. Gestational diabetes mellitus. Journal of Clinical Investigation 2005115 485-491. (doi:10.1172/ JCI24531)

10 Plowman GD, Green JM, Neubauer MG, Buckley SD, McDonald VL, Todaro GJ \& Shoyab M. The epithelin precursor encodes two proteins with opposing activities on epithelial cell growth. Journal of Biological Chemistry 1992267 13073-13078.

11 Stubert J, Richter DU, Gerber B \& Briese V. Expression pattern of progranulin in the human placenta and its effect on cell proliferation in the choriocarcinoma cell line BeWo. Journal of Reproduction and Development 201157 229-235. (doi:10.1262/ jrd.10-073K)

12 Katz A, Nambi SS, Mather K, Baron AD, Follmann DA, Sullivan G \& Quon MJ. Quantitative insulin sensitivity check index: a simple, accurate method for assessing insulin sensitivity in humans. Journal of Clinical Endocrinology and Metabolism $2000 \mathbf{8 5}$ 2402-2410. (doi:10.1210/jc.85.7.2402)

13 Mari A, Pacini G, Murphy E, Ludvik B \& Nolan JJ. A model-based method for assessing insulin sensitivity from the oral glucose tolerance test. Diabetes Care 200124 539-548. (doi:10.2337/ diacare.24.3.539)

14 Thomaseth K, Kautzky-Willer A, Ludvik B, Prager R \& Pacini G. Integrated mathematical model to assess $\beta$-cell activity during the oral glucose test. American Journal of Physiology $1996 \mathbf{2 7 0}$ E522-E531.
15 Winhofer Y, Handisurya A, Tura A, Bittighofer C, Klein K, Schneider B, Bieglmayer C, Wagner OF, Pacini G, Luger A \& Kautzky-Willer A. Osteocalcin is related to enhanced insulin secretion in gestational diabetes mellitus. Diabetes Care 201033 39-43. (doi:10.2337/dc09-1237)

16 Ahren B \& Pacini G. Impaired adaptation of first-phase insulin secretion in postmenopausal women with glucose intolerance. American Journal of Physiology 1997273 E701-E707.

$17 \mathrm{Lu} \mathrm{R} \&$ Serrero G. Mediation of estrogen mitogenic effect in human breast cancer MCF-7 cells by PC-cell-derived growth factor (PCDGF/granulin precursor). PNAS 200198 142-147. (doi:10.1073/pnas.011525198)

18 Suzuki M, Yonezawa T, Fujioka H, Matuamuro M \& Nishihara M. Induction of granulin precursor gene expression by estrogen treatment in neonatal rat hypothalamus. Neuroscience Letters 2001297 199-202. (doi:10.1016/S0304-3940(00)01699-2)

19 Chiba S, Suzuki M, Yamanouchi K \& Nishihara M. Involvement of granulin in estrogen-induced neurogenesis in the adult rat hippocampus. Journal of Reproduction and Development $2007 \mathbf{5 3}$ 297-307. (doi:10.1262/jrd.18108)

20 Desmarais JA, Cao M, Bateman A \& Murphy BD. Spatiotemporal expression pattern of progranulin in embryo implantation and placenta formation suggests a role in cell proliferation, remodeling, and angiogenesis. Reproduction 2008136 247-257. (doi:10.1530/REP-08-0044)

21 Esposito K, Nappo F, Marfella R, Giugliano G, Giugliano F, Ciotola M, Quagliaro L, Ceriello A \& Giugliano D. Inflammatory cytokine concentrations are acutely increased by hyperglycemia in humans: role of oxidative stress. Circulation $2002 \mathbf{1 0 6}$ 2067-2072. (doi:10.1161/01.CIR.0000034509.14906.AE)

22 Tonjes A, Fasshauer M, Kratzsch J, Stumvoll M \& Bluher M. Adipokine pattern in subjects with impaired fasting glucose and impaired glucose tolerance in comparison to normal glucose tolerance and diabetes. PLoS ONE 20105 e13911. (doi:10.1371/ journal.pone.0013911)

23 Buchanan TA, Metzger BE, Freinkel N \& Bergman RN. Insulin sensitivity and B-cell responsiveness to glucose during late pregnancy in lean and moderately obese women with normal glucose tolerance or mild gestational diabetes. American Journal of Obstetrics and Gynecology $19901621008-1014$.

24 Catalano PM, Huston L, Amini SB \& Kalhan SC. Longitudinal changes in glucose metabolism during pregnancy in obese women with normal glucose tolerance and gestational diabetes mellitus. American Journal of Obstetrics and Gynecology 1999180 903-916. (doi:10.1016/SO002-9378(99)70662-9)

25 Kirwan JP, Huston-Presley L, Kalhan SC \& Catalano PM. Clinically useful estimates of insulin sensitivity during pregnancy: validation studies in women with normal glucose tolerance and gestational diabetes mellitus. Diabetes Care 200124 1602-1607. (doi:10.2337/diacare.24.9.1602)

Received 23 January 2012

Revised version received 23 June 2012

Accepted 16 July 2012 